\title{
Magnetization dynamics of electron-impurity systems at paramagnetic resonance
}

\author{
E. A. Ivanchenko \\ National Science Center «Kharkov Institute of Physics and Technology», \\ Institute for Theoretical Physics, 1 Akademicheskaya Str., 61108 Kharkov, Ukraine \\ E-mail: yevgeny@kipt.kharkov.ua
}

Received September 27, 2001

\begin{abstract}
The equation for the magnetization is obtained on the basis of the kinetic equation for an isotropic distribution function of electrons scattering on massive impurity centers in the presence of magnetic and electric fields. The analytical solution of the Cauchy problem for a given initial distribution of the magnetization under conditions of paramagnetic resonance is obtained. The exact solutions are found for magnetization dynamics in samples having the forms of an ellipsoid of revolution and a cylinder, as well. The influence of magnetic exchange on the surface of the cylinder (III boundary value problem) is taken into account. The dependence of the magnetization on the static electric field is exponentially decreasing with time, with an exponent proportional to the electric field squared times the diffusion coefficient. For a fixed instant of time the magnetization depends nonlocally on the magnitude and direction of the electric field. The estimated dynamic shift of the forced precession has a nonlocal and nonlinear dependence on the nonuniform distribution of the initial magnetization. This shift is estimated with boundary conditions taken into account and is caused by a field similar to the Suhl-Nakamura field in a paramagnetic medium. The dynamic shift of the free precession has only a nonlocal character. The time and space dependence of the internal field is obtained. All results are expressed in terms of the initial distribution of the magnetization without specifying its functional form and in terms of the propagation function, which depends on the shape of the sample. These results may be used for analysis of spin diffusion in natural and manmade materials and also in magnetometry.
\end{abstract}

PACS: 33.35.+r, 75.40.Gb, 76.30.-v, 76.60.Jx, 76.60.-k

\section{Introduction}

A system of electrons, interacting among themselves and with motionless potential impurity centers randomly distributed in uniform external fields is described by a distribution function $f$ obeying the kinetic equation $[1,2]$

$$
\begin{gathered}
\frac{\partial}{\partial t} f+i[w, f]_{-}+\mathbf{v} \frac{\partial}{\partial \mathbf{x}} f+e \mathbf{E} \frac{\partial}{\partial \mathbf{p}} f+ \\
+\frac{q}{c}[\mathbf{v}, \mathbf{B}] \frac{\partial}{\partial \mathbf{p}} f=L f+L^{e e} f,
\end{gathered}
$$

where $f \equiv f_{\mathbf{p}}(\mathbf{x}, t)$ is the distribution function of the electrons, which is a matrix in the electron spin space; $q$ and $\mathbf{v}=\partial e_{\mathbf{p}} / \partial \mathbf{p}$ are the electron charge and velocity, respectively; $L$ and $L^{e e}$ are the electronimpurity and electron-electron collision integrals; $\mathbf{B}$ is the magnetic field, $\mathbf{E}$ is the static electric field; $w=-\mu_{0} \boldsymbol{\sigma} \mathbf{B}\left(\mu_{0}\right.$ is the Bohr magneton and $\boldsymbol{\sigma}$ are the Pauli matrices). We assume that massive charged impurities, whose kinetics is not considered here, form a neutralizing electrical background.

We shall define the distribution function of electrons over energy $e$ [3]

$$
\begin{gathered}
n(e, \mathbf{x}, t)=\langle f\rangle=\frac{1}{\rho(e)} \int d V_{\mathbf{p}} f_{\mathbf{p}}(\mathbf{x}, t) \delta\left(e-e_{\mathbf{p}}\right), \\
d V_{\mathbf{p}}=\frac{d^{3} p}{(2 \pi)^{3}},
\end{gathered}
$$


where

$$
\rho(e)=\int d V_{\mathbf{p}} \delta\left(e-e_{\mathbf{p}}\right)
$$

is the electron density of states, and the brackets mean the averaging defined by formula (2). It follows from Eq. (2) that $\langle\bar{L} f\rangle=0$, where

$$
\bar{L}=L+L^{\prime}(\mathbf{B}), \quad L^{\prime}(\mathbf{B})=-\frac{q}{c}[\mathbf{v}, \mathbf{B}] \frac{\partial}{\partial \mathbf{p}} .
$$

Indeed, the electron-impurity collision integral has the form

$$
(L f)(\mathbf{p})=2 \pi N \int d V_{\mathbf{p}^{\prime}} w\left(\mathbf{p}, \mathbf{p}^{\prime}\right) \delta\left(e_{\mathbf{p}}-e_{\mathbf{p}^{\prime}}\right)\left(f_{\mathbf{p}^{\prime}}-f_{\mathbf{p}}\right),
$$

and hence $\langle L f\rangle=0$. Here $N$ is the impurity density, $w\left(\mathbf{p}, \mathbf{p}^{\prime}\right)$ is the probability per unit time of electron scattering on the impurity center. As $\varepsilon_{i k l}\left(\partial v_{k} / \partial p_{i}\right)=\varepsilon_{i k l}\left(\partial^{2} e_{\mathbf{p}} / \partial p_{i} p_{k}\right)=0$, hence the mean

$$
\left\langle[\mathbf{v}, \mathbf{B}]\left(\frac{\partial}{\partial \mathbf{p}} f\right)\right\rangle=\varepsilon_{i k l} \frac{1}{\rho(e)} \int d V_{\mathbf{p}} v_{k} B_{l}\left(\frac{\partial}{\partial p_{i}} f\right) \delta\left(e-e_{\mathbf{p}}\right),
$$

and after integration by parts we have $\langle\bar{L} f\rangle=0$.

The operator $\bar{L}$ has the property

$$
\bar{L}(\mathbf{B})=\bar{L}^{+}(-\mathbf{B})
$$

where + means the conjugate operation, defined by the formula $(x, y) \equiv\langle x, y\rangle$. By virtue of the definition of the operators $L$ and $L^{\prime}$, we have $(L x, y)=(x, L y)$, $\left(L^{\prime}(\mathbf{B}) x, y\right)=\left(x, L^{\prime}(-\mathbf{B}) y\right)$, i.e., Eq. (4) is valid.

As the result of averaging Eq. (1), we obtain the equation for the distribution function $n(e, \mathbf{x}, t)$ :

$\frac{\partial}{\partial t} n+i[w, n]_{-}=\left\langle L^{e e} f\right\rangle-\frac{\partial}{\partial x_{k}} j_{k}-q E_{k} \frac{1}{\rho(e)} \frac{\partial}{\partial e}\left(\rho(e) j_{k}\right)$.

To close this equation one has to express the current $j_{k}$ in terms of $n$. This can be done, if the frequency of electron-impurity collisions $\tau_{e \text { imp }}^{-1}$ is much greater than the frequencies of electron-electron collisions $\tau_{e e}^{-1}$ and if the times $t$ are large in comparison with the corresponding relaxation time $\tau_{e \text { imp }}$, the electron distribution function becomes some functional of $n$, i.e., the electron distribution function becomes independent of the electron momentum direction due to the collisions of electrons with impurities. On this basis it is possible to show that in the linear approximation with respect to the gradients and electric field the diffusion current is [4]

$$
j_{k}=-D_{k i}(\mathbf{B})\left(\frac{\partial}{\partial x_{i}} n+q E_{i} \frac{\partial}{\partial e} n\right)
$$

where $D_{k i}(\mathbf{B})=\left\langle\bar{L}^{-1} v_{k}, v_{i}\right\rangle$ is the diffusion coefficient of electrons in a magnetic field having the property $D_{k i}(\mathbf{B})=D_{i k}(-\mathbf{B})$, which follows from (4).

In case of an isotropic electron dispersion relation $e_{\mathbf{p}}=e_{|\mathbf{p}|}$ we get

$$
\begin{aligned}
& D_{k i}=d\left[\delta_{k i}+b_{k} b_{i} \omega_{c}^{2} \tau_{e \text { imp }}+\varepsilon_{i k l} b_{i} \omega_{c}\right], \\
& d=\frac{\tau_{e \text { imp }} \mathbf{v}^{2}}{3\left(1+\omega_{c}^{2}\right)}, \quad \mathbf{b}=\frac{\mathbf{B}}{|\mathbf{B}|}, \quad \omega_{c}=\tau_{e \text { imp }} \Omega_{c} .
\end{aligned}
$$

The cyclotron frequency $\Omega_{c}$ is equal to $\Omega_{c}=(q|\mathbf{v}| B) / c|\mathbf{p}|$,

$$
\tau_{e \text { imp }}^{-1} \equiv 2 \pi N \int d V_{\mathbf{p}^{\prime}} w\left(\mathbf{p}, \mathbf{p}^{\prime}\right) \delta\left(e_{\mathbf{p}}-e_{\mathbf{p}^{\prime}}\right)\left(1-\frac{\mathbf{p p}^{\prime}}{\left|\mathbf{p} \mathbf{p}^{\prime}\right|}\right)
$$

Equations (5) and (6) together determine a closed equation for the distribution function $n(e, \mathbf{x}, t)$, which is isotropic with respect to the moments [4].

\section{Macroscopic equation for magnetization}

We define the macroscopic density of the electron magnetic moment $\mathbf{M}=\left(M_{1}, M_{2}, M_{3}\right)$, the magnetization, by the formula

$$
\mathbf{M}(\mathbf{x}, t)=2 \mu_{0} \operatorname{Sp} \frac{1}{2} \boldsymbol{\sigma} \int d V_{\mathbf{p}} n(e, \mathbf{x}, t)
$$

In view of the relation for Pauli matrixes $\sigma_{j} \sigma_{k}-\sigma_{k} \sigma_{j}=2 i \varepsilon_{j k l} \sigma_{l}$, the kinetic equation (5) after multiplication by $\mu_{0} \sigma$, taking the trace on the spin variables, and integration over $d V_{\mathbf{p}}$ takes the form

$$
\frac{\partial}{\partial t} \mathbf{M}_{i}+2 \mu_{0}[\mathbf{B}, \mathbf{M}]_{i}+\frac{\partial}{\partial x_{k}} I_{i k}=0
$$

where the flux density of the electron magnetic moment is equal to 


$$
I_{i k} \equiv-2 \mu_{0} \operatorname{Sp} \frac{1}{2} \sigma_{i} \int d V_{\mathbf{p}} D_{k p}\left(\frac{\partial n}{\partial x_{p}}+q E_{p} \frac{\partial n}{\partial e}\right) .
$$

In order to obtain a closed equation for the magnetization we assume that the function $D_{k p}$ is smooth over $e$, and it is therefore possible to take it out from under the integral sign. We integrate the second term in (11) by parts on $e$, and assume that the surface terms are small at $e=0$ and at $e=e_{F}$, where $e_{F}$ is the Fermi energy. This approximation allows us to write down the equation for the magnetization as

$\frac{\partial}{\partial t} \mathbf{M}+2 \mu_{0}[\mathbf{B}, \mathbf{M}]-D_{k p}\left(\frac{\partial^{2}}{\partial x_{k} \partial x_{p}}-\frac{e}{2 e_{F}} E_{p} \frac{\partial}{\partial x_{k}}\right) \mathbf{M}=0$.

Equation (12) without allowance for spatial inhomogeneity corresponds to the Bloch equation and forms the basis of the theory of paramagnetic resonance. The incorporation of inhomogeneity is carried out in Refs. 1, 5 without specifying the character of the diffusion mechanism. The nonlinear equation describing a collision dynamics of magnetization in the absence of external fields is obtained in Ref. 6 .

The purpose of the present work is to study the magnetization dynamics of electron-impurity systems on the basis of Eq. (12) under conditions of paramagnetic resonance with boundary conditions taken into account.

\section{Paramagnetic resonance in electron-impurity systems}

We consider the magnetization behavior in the case when the external magnetic field in Eq. (12) consists of two terms

$$
\mathbf{B}=\mathbf{B}_{0}+\mathbf{h}(t),
$$

where $\mathbf{B}_{0}$ is the static field and $\mathbf{h}(t)$ is the alternating field.

To find the solution of Eq. (12) we shall develop the scheme described by Bar'yakhtar and Ivanov in Ref. 7. For this purpose, we shall present the solution for the magnetization [see Eq. (12)] as an expansion in powers of the amplitude of the external alternating magnetic field:

$$
\mathbf{M}(\mathbf{x}, t)=\sum_{k=0}^{\infty} \mathbf{m}^{(k)}(\mathbf{x}, t)
$$

After substituting Eq. (13) into Eq. (12) we have an infinite system of equations for $\mathbf{m}^{(k)}$

$$
\begin{gathered}
\frac{\partial}{\partial t} \mathbf{m}^{(k)}+2 \mu_{0}\left[\mathbf{B}, \mathbf{m}^{(k)}\right]-D \mathbf{m}^{(k)}=-2 \mu_{0}\left[\mathbf{h}(t), \mathbf{m}^{(k-1)}\right] \\
k=0,1,2, \ldots ; \mathbf{m}^{(-1)} \equiv 0, \mathbf{B}=(0,0, B) \\
D \equiv d_{F}\left(\frac{\partial^{2}}{\partial x^{2}}+\frac{\partial^{2}}{\partial y^{2}}+\left(1+\omega_{c}^{2}\right) \frac{\partial^{2}}{\partial z^{2}}-\frac{q}{2 e_{F}}\left(E_{1}+\omega_{c} E_{2}\right) \frac{\partial}{\partial x}-\right. \\
\left.-\frac{q}{2 e_{F}}\left(E_{2}-\omega_{c} E_{1}\right) \frac{\partial}{\partial y}-\frac{q}{2 e_{F}} E_{3}\left(1+\omega_{c}^{2}\right) \frac{\partial}{\partial z}\right)
\end{gathered}
$$

At first we find the solution $\mathbf{m}^{(0)}$ of the Cauchy problem with the help of the change of dependent variables

$$
\begin{gathered}
m_{i}^{(0)}(\mathbf{x}, t)=(2 \pi)^{-3 / 2} \int d^{3} k \mathrm{e}^{-i \mathbf{k x}} m_{i}^{(0)}(\mathbf{k}, t) \\
(i=1,2,3) .
\end{gathered}
$$

For the Fourier-components $\mathbf{m}_{i}^{(0)}(\mathbf{k}, t)$ we get a system of differential equations of first order in time. This system is easily solved. Carrying out the inverse transformation,

$$
m_{i}^{(0)}(\mathbf{k}, t)=(2 \pi)^{-3 / 2} \int d^{3} x^{\prime} \mathrm{e}^{i \mathbf{k} \mathbf{x}^{\prime}} m_{i}^{(0)}(\mathbf{x}, t)
$$

we find the solution for the magnetization in the form of free precession in constant fields,

$$
\mathbf{m}^{(0)}(\mathbf{x}, t)=\int d^{3} x^{\prime} g\left(t, \mathbf{x}^{\prime}, \mathbf{x}\right) \times
$$

$\times\left(m\left(\mathbf{x}^{\prime}\right) \cos \left(\Omega t+\varphi\left(\mathbf{x}^{\prime}\right)\right),-m\left(\mathbf{x}^{\prime}\right) \sin \left(\Omega t+\varphi\left(\mathbf{x}^{\prime}\right)\right), m_{3}\left(\mathbf{x}^{\prime}\right)\right)$

on the set of the known initial data of the form

$$
\mathbf{m}^{(0)}(\mathbf{x}, t=0)=\left(m(\mathbf{x}) \cos \varphi(\mathbf{x}),-m(\mathbf{x}) \sin \varphi(\mathbf{x}), m_{3}(\mathbf{x})\right)
$$

with a propagation function equal to 


$$
\begin{gathered}
g\left(t, \mathbf{x}^{\prime}, \mathbf{x}\right)=\frac{1}{\left(1+\omega_{c}^{2}\right)^{1 / 2}}\left(\frac{1}{2\left(\pi d_{F} t\right)^{1 / 2}}\right)^{3} \exp \left[-\left(\frac{x^{\prime}-x}{2 \sqrt{d_{F} t}}+\frac{q}{2 e_{F}} \frac{E_{1}+\omega_{c} E_{2}}{2} \sqrt{d_{F} t}\right)^{2}-\right. \\
\left.-\left(\frac{y^{\prime}-y}{2 \sqrt{d_{F} t}}+\frac{q}{2 e_{F}} \frac{E_{2}-\omega_{c} E_{1}}{2} \sqrt{d_{F} t}\right)^{2}-\left(\frac{z^{\prime}-z}{2\left(1+\omega_{c}^{2}\right)^{1 / 2} \sqrt{d_{F} t}}+\frac{q}{2 e_{F}} \frac{\left(1+\omega_{c}^{2}\right)^{1 / 2} E_{3}}{2} \sqrt{d_{F} t}\right)^{2}\right], \\
\Omega=2 \mu_{0} B, \quad d_{F}=\frac{v_{F}^{2} \tau_{e \text { imp }}}{3\left(1+\omega_{c}^{2}\right)},
\end{gathered}
$$

where $v_{F}$ is the Fermi velosity.

This function obeys the equation

$$
\frac{\partial}{\partial t} g-D g=0
$$

and also has the properties

$$
\begin{gathered}
\lim _{t \rightarrow 0^{+}} g\left(t, \mathbf{x}^{\prime}, \mathbf{x}\right)=\delta\left(\mathbf{x}^{\prime}-\mathbf{x}\right), \\
\lim _{t \rightarrow 0^{+}} \frac{\partial}{\partial t} g\left(t, \mathbf{x}^{\prime}, \mathbf{x}\right)=D \delta\left(\mathbf{x}^{\prime}-\mathbf{x}\right) .
\end{gathered}
$$

An important property of the propagation function is that it satisfies the Smolukhowski-Chapman-Kolmogoroff equation

$$
\int d^{3} x^{\prime} g\left(t-t^{\prime}, \mathbf{x}^{\prime}, \mathbf{x}\right) g\left(t^{\prime}, \mathbf{x}^{\prime \prime}, \mathbf{x}\right)=g\left(t, \mathbf{x}^{\prime \prime}, \mathbf{x}\right)
$$

The particular solution $\mathbf{m}^{(1)}$ of Eq. (14) with the right-hand side $-2 \mu_{0}\left[\mathbf{h}(t), \mathbf{m}^{(0)}(\mathbf{x}, t)\right]$ can be obtained, using the semigroup property of the function $g\left(t, \mathbf{x}^{\prime}, \mathbf{x}\right)(24)$ :

$$
\begin{aligned}
m_{1}^{(1)}(\mathbf{x}, t)+i m_{2}^{(1)}(\mathbf{x}, t) & =\int_{0}^{t} d t^{\prime} \mathrm{e}^{i \Omega\left(t^{\prime}-t\right)} \int d^{3} x^{\prime} g\left(t, \mathbf{x}^{\prime}, \mathbf{x}\right)\left[\left(h_{1}\left(t^{\prime}\right)+i h_{2}\left(t^{\prime}\right)\right) m_{3}\left(\mathbf{x}^{\prime}\right)-h_{3}\left(t^{\prime}\right) \mathrm{e}^{-i\left(\Omega t^{\prime}+\varphi\left(x^{\prime}\right)\right)} m\left(\mathbf{x}^{\prime}\right)\right] \\
m_{3}^{(1)}(\mathbf{x}, t)= & \int_{0}^{t} d t^{\prime} \int d^{3} x^{\prime} g\left(t, \mathbf{x}^{\prime}, \mathbf{x}\right) m\left(\mathbf{x}^{\prime}\right)\left[h_{1}\left(t^{\prime}\right) \sin \left(\Omega t^{\prime}+\varphi\left(\mathbf{x}^{\prime}\right)\right)+h_{2}\left(t^{\prime}\right) \cos \left(\Omega t^{\prime}+\varphi\left(\mathbf{x}^{\prime}\right)\right)\right]
\end{aligned}
$$

It is seen from formulas (25), (26), that the magnetization at the time $t$ is determined by the field $\mathbf{h}(t)$ at all previous times, starting at the instant it is turned on.

We choose left rotation for the external alternating magnetic field, which is perpendicular to the static field $\mathbf{B}_{0}, \mathbf{h}(t)=h(\cos \omega t,-\sin \omega t, 0), h$ is the amplitude of the field, and $\omega$ is the frequency of the alternating magnetic field. Since at the paramag- netic resonance $\omega=\Omega$, we find from formulas (25), (26) that the particular solution for the magnetization in the approximation linear in the field is the forced precession

$$
\begin{gathered}
m_{1}^{(1)}(\mathbf{x}, t)=\omega_{1} t m_{3}^{(0)}(\mathbf{x}, t) \cos (\Omega t-\pi / 2), \\
m_{2}^{(1)}(\mathbf{x}, t)=-\omega_{1} t m_{3}^{(0)}(\mathbf{x}, t) \sin (\Omega t-\pi / 2),
\end{gathered}
$$




$$
m_{3}^{(1)}(\mathbf{x}, t)=\omega_{1} t A(\mathbf{x}, t)
$$

$A(\mathbf{x}, t) \equiv \int d^{3} x^{\prime} g\left(t, \mathbf{x}^{\prime}, \mathbf{x}\right) m\left(\mathbf{x}^{\prime}\right) \sin \varphi\left(\mathbf{x}^{\prime}\right), \omega_{1}=2 \mu_{0} h$,

lagging in phase behind the phase of the alternating magnetic field by $\pi / 2$.

Having continued the procedure of iteration, we can sum the series on $\omega_{1} t$ and find the general exact solution for the magnetization dynamics (12) at paramagnetic resonance:

$$
\begin{gathered}
\mathbf{M}(\mathbf{x}, t)=\mathbf{m}^{(0)}(\mathbf{x}, t)+\mathbf{m}(\mathbf{x}, t), \\
\mathbf{m}(\mathbf{x}, t)= \\
=\left(\left[A(\mathbf{x}, t)\left(1-\cos \omega_{1} t\right)+m_{3}^{(0)}(\mathbf{x}, t) \sin \omega_{1} t\right] \sin \Omega t,\right. \\
{\left[A(\mathbf{x}, t)\left(1-\cos \omega_{1} t\right)+m_{3}^{(0)}(\mathbf{x}, t) \sin \omega_{1} t\right] \cos \Omega t,} \\
\left.A(\mathbf{x}, t) \sin \omega_{1} t+m_{3}^{(0)}(\mathbf{x}, t)\left(\cos \omega_{1} t-1\right)\right) .
\end{gathered}
$$

At $h=0$ this solution transforms into $\mathbf{M}(\mathbf{x}, t)=$ $=\mathbf{m}^{(0)}(\mathbf{x}, t)$; see Eq. (18). As is seen from the solution (29), there is no divergence in time. Finally we come to the conclusion that the solution of the Cauchy problem of Eq. (12) with the initial distribution (19) in the class of square-integrable functions is completely determined by the propagation function and the shape of the sample, i.e., by the integration volume. For an unbounded medium under conditions of paramagnetic resonance the solution of the Cauchy problem takes the form of Eq. (29).

The magnetization projection $M_{3}(\mathbf{x}, t)$ oscillates. This fact implies that a population inversion occurs in the system considered.

For the analysis of the forced precession we write the solution (29) for $M_{1}, M_{2}$ as

$$
\begin{gathered}
M_{1}(\mathbf{x}, t)=a \cos (\Omega t+\phi), \\
M_{2}(\mathbf{x}, t)=-a \sin (\Omega t+\phi),
\end{gathered}
$$

where the local amplitude and phase of precession are equal to

$$
\begin{gathered}
a(\mathbf{x}, t)= \\
=\sqrt{\left[-A(\mathbf{x}, t) \cos \omega_{1} t+m_{3}^{(0)}(\mathbf{x}, t) \sin \omega_{1} t\right]^{2}+A_{1}^{2}(\mathbf{x}, t)},
\end{gathered}
$$

$$
\begin{gathered}
\phi(\mathbf{x}, t)=\operatorname{arctg} \frac{A(\mathbf{x}, t) \cos \omega_{1} t-m_{3}^{(0)}(\mathbf{x}, t) \sin \omega_{1} t}{A_{1}(\mathbf{x}, t)}, \\
A_{1}(\mathbf{x}, t) \equiv \int d^{3} x^{\prime} g\left(t, \mathbf{x}^{\prime}, \mathbf{x}\right) m\left(\mathbf{x}^{\prime}\right) \cos \varphi\left(\mathbf{x}^{\prime}\right) .
\end{gathered}
$$

Expanding the phase $\phi(\mathbf{x}, t)$ (31) in a series with respect to $t$ and restricting ourselves to the term linear in $t$, we get, in view of the property (23), the local dynamic shift of the forced frequency $\Omega^{\prime}$ with respect to the Larmor precession frequency $\Omega$,

$$
\begin{gathered}
\phi(\mathbf{x}, t)=\varphi(\mathbf{x})+\Omega^{\prime}(\mathbf{x}, 0) t+\ldots, \\
\Omega^{\prime}(\mathbf{x}, 0)=\frac{1}{m^{2}(\mathbf{x})}\left\{\left[-\omega_{1} m_{3}(\mathbf{x})+\right.\right. \\
\left.+\int d^{3} x^{\prime}\left(D \delta\left(\mathbf{x}-\mathbf{x}^{\prime}\right)\right) m\left(\mathbf{x}^{\prime}\right) \sin \varphi\left(\mathbf{x}^{\prime}\right)\right] m(\mathbf{x}) \cos \varphi(\mathbf{x})- \\
-\left[\int d^{3} x^{\prime}\left(D \delta\left(\mathbf{x}-\mathbf{x}^{\prime}\right)\right) m\left(\mathbf{x}^{\prime}\right) \cos \varphi\left(\mathbf{x}^{\prime}\right)\right] \times \\
\left.\times\left(m(\mathbf{x}) \sin \varphi(\mathbf{x})-\omega_{1} m_{3}(\mathbf{x})\right)\right\},
\end{gathered}
$$

the cause of which has the meaning of the internal field at the point $\mathbf{x}$ (an analog of the Suhl-Nakamura field [8] in a paramagnetic medium, coordinated with the boundary conditions). This field depends on an initial nonuniform magnetization distribution at all points of the sample and the shape of the sample, that is, it has a nonlocal character. Without nonlocality being taken into account, this shift is proportional to the amplitude of the forced field and has the simple form,

$$
\Omega^{\prime}(\mathbf{x}, 0)=-2 \mu_{0} h \frac{m_{3}(\mathbf{x}) \cos \varphi(\mathbf{x})}{m(\mathbf{x})} .
$$

As it is seen from the formula (33), this shift depends nonlinearly on the initial distribution. This result coincides with that of Ref. 8 in view of heterogeneity. It follows from formula (32) that the dynamic shift of the free precession $\Omega_{\text {free }}^{\prime}(\mathbf{x}, 0)$ is completely nonlocal:

$$
\Omega_{\text {free }}^{\prime}(\mathbf{x}, 0)=
$$

$$
=-\frac{1}{m(\mathbf{x})} \int d^{3} x^{\prime}\left(D \delta\left(\mathbf{x}-\mathbf{x}^{\prime}\right)\right) m\left(\mathbf{x}^{\prime}\right) \sin \left(\varphi(\mathbf{x})-\varphi\left(\mathbf{x}^{\prime}\right)\right) .
$$


Now it is obvious that in the general case the dependence of the dynamic shift on time and coordinates is

$$
\Omega^{\prime}(\mathbf{x}, t)=\frac{\phi(\mathbf{x}, t)-\varphi(\mathbf{x})}{t} .
$$

We find the maximal amplitude of the forced precession $a^{\max }$ from the condition $M_{3}=0$, i.e.,

$$
A \sin \omega_{1} t+m_{3}^{(0)} \cos \omega_{1} t=0 .
$$

After substituting (36) in (31), we get

$$
a^{\max }=\left(\frac{m_{3}^{(0)^{2}}}{\sin ^{2} \omega_{1} t}+A_{1}^{2}\right)^{1 / 2},
$$

and the times $t^{(k)}$ are determined by the solution of Eq. (36), which can be written in equivalent form as

$$
\sin \left(\omega_{1} t+\delta\right)=0, \quad \delta=\operatorname{arctg} \frac{m_{3}^{(0)}}{A} .
$$

In the simplest case we find $\delta \approx-\pi / 4, \omega_{1} t \approx k \pi+$ $+\pi / 4, k=0,1,2, \ldots$

$$
\begin{gathered}
t^{(0)} \approx \frac{\pi}{4 \omega_{1}}, t^{(1)} \approx \frac{5 \pi}{4 \omega_{1}}, \ldots, \\
a_{t=t^{(0)}}^{\max } \approx \sqrt{3}\left|m_{3}^{(0)}\right|_{t=t^{(0)}} .
\end{gathered}
$$

Decaying bursts of precession amplitude $a^{\max }$ are observed.

To take into account the particular shape of the sample it is necessary to use the eigenfunctions of the Laplace operator in cylindrical, spherical, or other coordinates as well. The general formulas (29) and the ones related to them retain their form under replacement of the propagation function and volume of integration. The propagation functions for the cylinder and ellipsoid of revolution are given in Appendix 1. The general exact solution of the equation (12) is given in Appendix 2.

\section{Conclusions}

The dynamics of the evolution of a system of electrons and impurities placed in static electric and magnetic fields is investigated under the influence of an alternating magnetic field under conditions of paramagnetic resonance. The general formulas for all three magnetization components in their evolutionary interrelation are obtained with the shape of the sample (cylinder, ellipsoid of revolution) taken into account, since experimental engineering allows one to measure these components [9]. The behavior of forced precession in samples is theoretically investigated. The dynamic shift of the frequency of paramagnetic resonance caused by a nonuniform distribution of initial magnetization is found. All results are expressed in terms of the initial magnetization distribution and a propagation function dependent on the shape of the sample. The results obtained are applied to the analysis of spin diffusion in natural and manmade materials [10,11] and also in magnetometry [9].

\section{Acknowledgements}

The author thanks Prof. S. V. Peletminskii for interest in the research and for useful discussion.

\section{Appendix 1}

Cylinder (III boundary value problem) Ref. 12

$$
\begin{gathered}
\mathbf{E}=\left(0,0, E_{3}\right), \\
x=r \cos \varphi, \quad y=r \sin \varphi, \frac{z}{\sqrt{1+\omega_{c}^{2}}}=u ; \\
0 \leq r \leq r_{0}, \quad 0 \leq \varphi \leq 2 \pi, \quad 0 \leq z \leq l, \quad 0 \leq t \leq \infty, \\
d^{3} x^{\prime}=r^{\prime} d r^{\prime} d \varphi^{\prime} d u^{\prime} .
\end{gathered}
$$

The boundary conditions are

$$
\begin{aligned}
& {\left[\frac{\partial}{\partial z} \mathbf{M}-h_{1} \mathbf{M}\right]_{z=0}=0} \\
& {\left[\frac{\partial}{\partial z} \mathbf{M}+h_{2} \mathbf{M}\right]_{z=l}=0} \\
& {\left[\frac{\partial}{\partial r} \mathbf{M}+H_{3} \mathbf{M}\right]_{z=l}=0}
\end{aligned}
$$

where $h_{1}, h_{2}, H_{3}$ are the surface magneto-exchange factors.

The initial conditions are

$$
\mathbf{M}_{t=0}=\left(m(\mathbf{x}) \cos \varphi(\mathbf{x}),-m(\mathbf{x}) \sin \varphi(\mathbf{x}), m_{3}(\mathbf{x})\right) .
$$

The propagation function is 


$$
\begin{gathered}
g\left(t, r^{\prime}, \varphi^{\prime}, u^{\prime}, r, \varphi, u\right)=\frac{4}{\pi r_{0}^{2}} \exp \left(-\frac{q E_{3}}{4 e_{F}} \sqrt{1+\omega_{c}^{2}}\left(u^{\prime}-u\right)-\frac{\left(1+\omega_{c}^{2}\right) q^{2} E_{3}^{2}}{16 e_{F}^{2}} d_{F} t\right) \times \\
\left.\times \sum_{\substack{n=0 ; m, k=1 \\
\infty}}^{\infty} l+\frac{\left(H_{1} H_{2}+v_{m}^{2}\right)\left(H_{1}+H_{2}\right)}{\left(H_{1}^{2}+v_{m}^{2}\right)\left(H_{2}^{2}+v_{m}^{2}\right)}\right]^{-1}\left(1+\frac{r_{0}^{2} H_{3}-n^{2}}{\left(\mu_{k}^{(n)}\right)^{2}}\right)^{-1} J_{n}^{-2}\left(\mu_{k}^{(n)}\right) J_{n}\left(\frac{\mu_{k}^{(n)}}{r_{0}} r\right) J_{n}\left(\frac{\mu_{k}^{(n)}}{r_{0}} r^{\prime}\right) \times \\
\times \exp \left(-\left[\left(\frac{\mu_{k}^{(n)}}{r_{0}}\right)^{2}+v_{m}^{2}\right] d_{F} t\right)\left(\varepsilon_{n}^{-1} \cos n \varphi \cos n \varphi^{\prime}+\sin n \varphi \sin n \varphi^{\prime}\right) \sin \left(v_{m} u+z_{m}\right) \sin \left(v_{m} u^{\prime}+z_{m}\right),
\end{gathered}
$$

$\varepsilon_{n}=2$ if $n=0$ and $\varepsilon_{n}=1$ if $n \neq 0, J_{n}$ are the Bessel functions, $\mu_{k}^{(n)}, v_{m}$ are the positive roots according to the equations

$$
\begin{gathered}
\mu_{k}^{(n)} J^{\prime}\left(\mu_{k}^{(n)}\right)+r_{0} H_{3} J\left(\mu_{k}^{(n)}\right)=0, \quad \operatorname{ctg} \frac{v_{m} l}{\sqrt{1+\omega_{c}^{2}}}=\frac{v_{m}^{2}-H_{1} H_{2}}{v_{m}\left(H_{1}+H_{2}\right)}, \\
z_{m}=\operatorname{arctg} \frac{v_{m}}{H_{1}}, \quad H_{1}=\sqrt{1+\omega_{c}^{2}}\left(h_{1}-\frac{q E_{3}}{4 e_{F}}\right), \quad H_{2}=\sqrt{1+\omega_{c}^{2}}\left(h_{2}+\frac{q E_{3}}{4 e_{F}}\right) .
\end{gathered}
$$

\section{Ellipsoid of revolution}

$$
\begin{gathered}
x=r \cos \theta \sin \varphi, \quad y=r \cos \theta \cos \varphi, \quad u=\frac{z}{\sqrt{1+\omega_{c}^{2}}}=r \cos \theta, \\
0 \leq r \leq r_{0}, \quad 0 \leq \varphi \leq 2 \pi, \quad 0 \leq \theta \leq \pi, \quad d^{3} x^{\prime}=r^{2} \sin \theta^{\prime} d r^{\prime} d \theta^{\prime} d \varphi^{\prime} .
\end{gathered}
$$

The boundary conditions are $[\mathbf{M}(\mathbf{x}, t)]_{r=r_{0}}=0$.

The initial conditions are $\mathbf{M}_{t=0}=\left(m(\mathbf{x}) \cos \varphi(\mathbf{x}),-m(\mathbf{x}) \sin \varphi(\mathbf{x}), m_{3}(\mathbf{x})\right)$. The propagation function is

$$
\begin{gathered}
g\left(t, r^{\prime}, \theta^{\prime}, \varphi^{\prime}, r, \theta, \varphi\right)= \\
=\frac{1}{\pi r_{0}^{2}} \exp \left(-\frac{q\left(E_{1}+\omega_{c} E_{2}\right)\left(x^{\prime}-x\right)}{4 e_{F}}-\frac{q\left(E_{2}-\omega_{c} E_{1}\right)\left(y^{\prime}-y\right)}{4 e_{F}}-\frac{q E_{3} \sqrt{1+\omega_{c}^{2}}\left(u^{\prime}-u\right)}{4 e_{F}}-\frac{\left(1+\omega_{c}^{2}\right) q^{2} \mathbf{E}^{2} d_{F} t}{16 e_{F}^{2}}\right) \times \\
\times \sum_{n=0, m=1}^{\infty} \sum_{k=1}^{n} \frac{\varepsilon_{k}^{-1} \cos k \varphi \cos k \varphi^{\prime}+\sin k \varphi \sin k \varphi^{\prime}}{\frac{(n+k) !}{(2 n+1)(n-k) !}\left[J^{\prime}{ }_{n+1 / 2}\left(\mu_{m}^{(n)}\right)\right]^{2}} \exp \left(-\left(\mu_{m}^{(n)} / r_{0}\right)^{2} d_{F} t\right) \times \\
\times \frac{J_{n+1 / 2}\left(\mu_{m}^{(n)} r / r_{0}\right) J_{n+1 / 2}\left(\mu_{m}^{(n)} r^{\prime} / r_{0}\right)}{\sqrt{r r^{\prime}}} P_{n, k}(\cos \theta) P_{n, k}\left(\cos \theta^{\prime}\right),
\end{gathered}
$$

$J_{n+1 / 2}$ are the Bessel functions of half-integral order, $P_{n, k}$ are the associated Legendre functions, and $\mu_{m}^{(n)}$ are the positive roots of the equation $J_{n+1 / 2}\left(\mu_{m}^{(n)}\right)=0$. 


\section{Appendix 2}

If the mismatch $\Delta=\Omega-\omega$, i.e. the difference between Larmor precession $\Omega$ and the frequency of the alternating magnetic field is not equal to zero, the general exact solution of the equation (12) has the form:

$$
\begin{gathered}
\mathbf{M}(\mathbf{x}, \Delta, t)=\mathbf{m}^{(0)}(\mathbf{x}, t)+\mathbf{m}(\mathbf{x}, \Delta, t), \\
\mathbf{m}(\mathbf{x}, \Delta, t)=\left(\left(-A \cos \gamma t+\frac{\omega_{1}}{\gamma} m_{3}^{(0)} \sin \gamma t\right) \sin \omega t+A_{1} \cos \omega t-\frac{\Delta}{\gamma} A_{1} \sin \gamma t \sin \omega t-A_{1} \cos \Omega t+A \sin \Omega t-\right. \\
-\left(\frac{\Delta}{\gamma} A \sin \gamma t-\left(\omega_{1} \Delta m_{3}^{(0)}-\Delta^{2} A_{1}\right) \frac{1-\cos \gamma t}{\gamma^{2}}\right) \cos \omega t,\left(-A \cos \gamma t+\frac{\omega_{1}}{\gamma} m_{3}^{(0)} \sin \gamma t\right) \cos \omega t-A_{1} \sin \omega t+ \\
+\left(\frac{\Delta}{\gamma} A \sin \gamma t-\left(\omega_{1} \Delta m_{3}^{(0)}-\Delta^{2} A_{1}\right) \frac{1-\cos \gamma t}{\gamma^{2}}\right) \sin \omega t-\frac{\Delta}{\gamma} A_{1} \sin \gamma t \cos \omega t+A_{1} \sin \Omega t+A \cos \Omega t, \\
\left.m_{3}^{(0)}(\cos \gamma t-1)+\frac{\omega_{1}}{\gamma} A \sin \gamma t+\left(\Delta^{2} m_{3}^{(0)}+\omega_{1} \Delta A_{1}\right) \frac{1-\cos \gamma t}{\gamma^{2}}\right),
\end{gathered}
$$

where $\gamma=\sqrt{\omega_{1}^{2}+\Delta^{2}}$.

The dynamic shift is $\Omega^{\prime}(\mathbf{x}, \Delta, t)=\frac{\phi(\mathbf{x}, \Delta, t)-\varphi(\mathbf{x})}{t}$, where

$$
\phi(\mathbf{x}, \Delta, t)=\operatorname{arctg} \frac{A \cos \gamma t-\omega_{1} m_{3}^{(0)}(\sin \gamma t / \gamma)+\Delta A_{1}(\sin \gamma t / \gamma)}{A_{1}-\Delta A(\sin \gamma t / \gamma)+\left(\omega_{1} \Delta m_{3}^{(0)}-\Delta^{2} A_{1}\right)(1-\cos \gamma t) / \gamma^{2}}
$$

1. V. P. Silin, Sov. Phys. JETP 3, 305 (1956).

2. M. Ya. Azbel', V. I. Gerasimenko, and I. M. Lifshitz, Sov. Phys. JETP 5, 986 (1957).

3. L. V. Keldush, Sov. Phys. JETP 48, 1692 (1965).

4. E. A. Ivanchenko, V. V. Krasil'nikov, and S. V. Peletminskii, Fiz. Met. Metalloved. 57, 441 (1984) (in Russian).

5. G. D. Gaspari, Phys. Rev. 151, 215 (1966).

6. T. L. Andreeva and P. L. Rubin, Sov. Phys. JETP 91, $761(2000)$.

7. V. Baryakhtar and B. Ivanov, Modern magnetism, Nauka, Moscow (1986).
8. M. I. Kurkin and E. A. Turov, NMR $v$ magnitouporyadochnukh vetshestvakh $i$ yego primeneniye, Nauka, Moscow (1990), p. 148.

9. N. M. Pomerantsev, V. M. Ryzhov, and G. V. Skrotskii, Physicheskie osnovy kvantovoi magnitometrii, Nauka, Moscow (1972), p. 142.

10. K. R. Brownstein and C. E. Tarr, Phys. Rev. 19A, 2446 (1979).

11. Yi-Qiao Song, Phys. Rev. Lett. 85, 38 (2000).

12. B. M. Budak, A. A. Samarskii, and A. N. Tikhonov, Sbornik zadach po matematicheskoi physike, Nauka, Moscow (1980). 